\title{
Mechanical, Thermal, and Morphological Study of Nanoclay Reinforced Bio-based Poly Lactic Acid/Poly (3-hydroxy butyrate co-3-valerate) (PLA/PHBV) Blend
}

\author{
SMK Hasan ${ }^{1}$, MK Hossain ${ }^{2 *}$, SN Shaily², HJ Harrigan² and T Mickens ${ }^{2}$ \\ ${ }^{1}$ Department of Materials Science and Engineering, Tuskegee University, Tuskegee, Alabama, USA \\ ${ }^{2}$ Department of Mechanical Engineering, Tuskegee University, Tuskegee, Alabama, USA
}

*Corresponding author: MK Hossain, Department of Mechanical Engineering,

Tuskegee University, Tuskegee, Alabama, USA

Received Date: June 09, 2020

Published Date: July 24, 2020

\begin{abstract}
A hybrid biopolymer blend using PLA/PHBV with tailored material properties has been fabricated by using solvent casting method. Biopolymer blends of $25 / 75,50 / 50$, and $75 / 25$ wt.\% were investigated for property optimization. Afterwards, different percentages (1, 2, and 3 wt.\%) of nanoclay (NC) were incorporated into the optimized (75/25 wt.\%) system to further enhance the properties. DSC, TGA, Tensile, and SEM tests were conducted to investigate mechanical, thermal and morphological properties. It was observed that the crystallinity of the PLA phase increased with increasing amount of NC. Also, the tensile strength of PLA and PHBV film was found to be $29.20 \mathrm{MPa}$ and $16.79 \mathrm{MPa}$, respectively, whereas, $75 / 25$ wt.\% blend showed $25.73 \mathrm{MPa}$ which is higher than PHBV phase. Then, the addition of $2 \mathrm{wt} . \% \mathrm{NC}$ showed optimized and better mechanical and thermal properties compared to individual polymer phase. Also, two glass transition temperatures were found from thermal analysis which is the indication of the solution immiscibility. It was revealed from the SEM micrographs that the $2 \mathrm{wt} \% \mathrm{NC}$ was dispersed uniformly throughout the resin blend.
\end{abstract}

Keywords: Blend; PHBV; Nanoclay; DSC; TGA; SEM

Abbreviation: Blend Mixture of two macromolecular substances; DSC Differential Scanning Calorimetry; Nanoclay Layered Mineral Silicate; PHBV Poly-(3-hydroxy butyrate-co-3-valerate); PLA Poly-Lactic Acid; SEM Scanning Electron Microscopy; TGA Thermogravimetric Analysis; DTG Derivative Thermogravimetry

\section{Introduction}

In present age, the existence of human being on earth is threatened due to the proliferation of environmental pollution caused by plastic waste disposal and carbon-di-oxide emission. To solve this issue, researchers intend to use biodegradable and bio-based polymers instead of petroleum-based polymers which is the major source of plastic waste as well as carbon dioxide emission. In addition, increasing costs of petrochemical-based polymers and the demand of sustainable manufacturing have drawn attention of researchers towards biodegradable polymers [1]. A very common bio-based polymer is PLA. Ring opening polymerization of lactide, characterized by condensation polymerization of lactic acid mono

mer which is extracted from a renewable resource via. fermentation is the major source of PLA production. In its brief existence, lactide polymerization by ring opening has been widely used due to its short span of time, good mechanical properties, low cost, biocompatibility, easy disposal, and comparable thermoplasticity of petroleum-based polymers $[2,3]$. But, mechanical brittleness, low melting properties, slow crystallization, and low heat resistance have restricted its use to medical devices and the automobile industry [4-6]. Several techniques are available in literature to make its uses broader. Among those, researchers have recognized polymer blending as a most effective technique [7]. 
Another popular biodegradable polymer, poly (3-hydroxy butyrate-co-3-hydroxyvalerate) (PHBV), a member of the Polyhydroxyalkanoates (PHAs) family, is synthesized by microorganisms. It is a copolymer of Poly hydroxy butyrate (PHB) and Poly hydroxy valerate (PHV). In order to reduce the stiffness and crystallinity of PHB, copolymerization of PHB and PHV has been introduced by researchers [8]. PHBV is currently used in water filtration, food packaging, and biomedical applications. However, the disadvantages of PHBV are low crystallization rates, poor thermal stability, and lack of durability which hinders its use in large quantities [9]. In this regard, blending PLA and PHBV is a realistic approach to tailor the structure and properties without sacrificing biodegradability [10]. Several researchers have reported successful PLA/PHBV blend fabrication by using solvent casting method [11].

In most cases, polymers are thermodynamically immiscible due to the unfavorable interaction. But, PLA and PHBV polymers are partially miscible. Property combination of the polymer is essential for the enhancement of overall properties of the blend. However, both poor interfacial adhesion and macro - phase separation will restrict this combination [1]. To resolve this issue, researchers have introduced the concept of compatibilization by inorganic nanoparticle. But, the attempts to improve the interfacial bonding between the polymers by solid natural particles are very few [12-15].

There are many types of natural silicates, or fillers found such as mica, closite, montmorillonite, hectorite, and saponite [16-18]. By adding a small amount of NC into the composite, the mechanical, thermal, flame resistance, and moisture barrier properties can be improved. NC is a very popular filler for a composite material as it is environmentally friendly, inexpensive, and economical due to their natural sources [19]. By adding NC into the composite, the strain to failure decreases and the modulus of the composite increases. The degree of morphology and dispersion of the NC are vital, as the reinforcement of the fiber-matrix depends on it. To achieve the highest benefit NC should be exfoliated into the matrix. However, most of the time there is a combination of intercalated and exfoliated morphologies of $\mathrm{NC}$ into the polymer. Currently, processing techniques being used to disperse $\mathrm{NC}$ into polymer matrix include exfoliation-adsorption, in-situ polymerization, solution intercalation, and template synthesis. Due to the improper dispersion some nanoclay agglomerates into the composites. In order to remove larger clay which agglomerates in nanoclay suspension, a stabilization process was used in this study. In this research a hybrid biopolymer blend using PLA and PHBV was prepared. Different percentages $(1,2$, and $3 \mathrm{wt} \%)$ of nanoclay was added to the system using the solution intercalation technique to enhance mechanical and thermal properties of the polymer blend.

\section{Materials and Processing}

\section{Materials}

In this research, a commercial grade (PLA 2002D) polylactic acid (PLA) was purchased from Natureworks, LLC (USA). The PLA has density of $1.24 \mathrm{~g} / \mathrm{cm}^{3}, 4 \%$ D-isomer content, and 33\% crystallinity rate. Also, another biodegradable polymer poly (3-hydroxybutyrate-co-valerate) (PHBV), extracted from microorganism was supplied by Goodfellow Corporation, Coraopolis, PA, USA. It contains $12 \mathrm{~mol} \%$ valerite and has $150 \stackrel{\circ}{\circ} \mathrm{C}$ melting temperature, density of $1.25 \mathrm{~g} / \mathrm{cm}^{3}$, and $59 \%$ crystallinity rate. The commercial grade chloroform supplied by Sigma-Aldrich, USA was used. The nanoclay (Cloisite $\mathrm{Na}^{+}$), was obtained from Southern Clay Production, Inc., Texas, USA.

\section{Processing of polymer blends}

Net polymer blend: First, $180 \mathrm{ml}$ of chloroform was poured into a plastic beaker. Then, different percentages $(25 / 75,50 / 50$, $75 / 25)$ of PLA/PHBV blends were prepared. The ratio of PHBV and chloroform was maintained as 1:18 for all solutions. The weight of PLA was then taken according to the amount of PHBV as all the solvent is not suitable for dissolving PHBV polymer. The solution was stirred magnetically for 24 hours. Afterwards, the solution was vacuum mixed for 15 minutes using $100 \mathrm{kPa}$ pressure at a speed rate of $1500 \mathrm{r} / \mathrm{min}$ to remove the entrapped air bubbles. Finally, the solution was spread on a glass plate to cure for 24 hours and to remove the chloroform.

PLA/PHBV/NC blend: $180 \mathrm{ml}$ chloroform was poured into the plastic beaker. The $\mathrm{NC}$ was then poured into the solvent and ultrasonicated for 30 minutes at an amplitude of $45 \%$ with 10/10 on/off pulse rate. Then, 10 gm PHBV and 30 gm PLA was added into the solution and other steps were like neat polymer blend preparation.

\section{Experimental Procedure}

\section{Differential scanning calorimetry (DSC)}

Crystallization characteristic, melting temperature, melting enthalpy as well as degree of crystallinity were analyzed by TA instruments Q1000. Measurements were carried out in nitrogen atmosphere. Ten to twelve mg samples were heated from $30{ }^{\circ} \mathrm{C}$ to $200{ }^{\circ} \mathrm{C}$ with a heating rate of $10{ }^{\circ} \mathrm{C} / \mathrm{min}$. Then, the samples were held at $200{ }^{\circ} \mathrm{C}$ for 5 minutes to remove the prior thermal history. In addition, the samples were cooled to room temperature at the same rate and then again heated to temperature of $200{ }^{\circ} \mathrm{C}$. The degree of crystallinity was calculated using Eq. (1):

$$
X_{c}=\frac{\Delta H_{m}}{\Delta H_{m}^{o} f_{p}} \times 100 \%
$$

Where, $\Delta \mathrm{Hm}\left(\mathrm{Jg}^{-1}\right)$ indicates the melting enthalpy of polymer matrix, fp represents the polymer weight fraction in the sample, and $\Delta H_{m}^{o}\left(146 \mathrm{Jg}^{-1}\right)$ stands for the melting enthalpy of pure crystalline PHBV matrix.

\section{Thermogravimetric analysis (TGA)}

Thermal response of all the samples were recorded by TA Q500 analyzer. To analyze the thermal decomposition behavior, maxi- 
mum degradation temperature, and residual weight percentages of PLA/PHBV as well as PLA/PHBV/NC, ten to twelve mg samples were used. The samples were placed into a platinum pan under nitrogen atmosphere to heat up to $600{ }^{\circ} \mathrm{C}$ from room temperature at a heating rate of $10^{\circ} \mathrm{C} / \mathrm{min}$.

\section{Tensile test}

Tensile properties of all PLA/PHBV blends (25/75, 50/50 and $75 / 25$ wt\%) without NC and 75/25 wt\% PLA/PHBV with different amounts of NC (1, 2, and 3 wt\%) were carried out using a Zwick-Roell Z 2.5 testing unit according to the ASTM D882-02 standard. 1.2 $\mathrm{mm} / \mathrm{min}$ crosshead speed and $40 \mathrm{~mm}$ gauge length were maintained. 100x10x3 (mm x mm x mm) samples were tabbed between two aluminum grips.

\section{Morphological characterization}

Morphology of prepared specimens was studied by Olympus DP72 optical microscope with magnification from 7X to 115X.

Scanning Electron Microscopy (SEM) was also performed using a Hitachi S-3400N scanning electron microscope to observe proper dispersion of nanoclay inside the polymer blends. A $20 \mathrm{KV}$ accelerating voltage was applied to achieve the desired magnification.

\section{Result and Discussion}

\section{Differential scanning calorimetry (DSC)}

Figure 1(a) represents the DSC graph of different combinations of the PLA/PHBV blend with and without nanoclay. Figure 1(b,c) shows the heating and cooling scan separately. By the first heating scan from the molding, a thermal process was removed. After that, the second heating scan was performed by using same thermal process. From the heating scan, two melting temperatures $\mathrm{T}_{\mathrm{m} 1}$ and $\mathrm{T}_{\mathrm{m} 2}$ ), melting enthalpy, and from the cooling scan crystallization temperature $\left(\mathrm{T}_{\mathrm{c}}\right)$ was determined. Melting temperatures, crystallization temperature, melting enthalpy, degree of crystallinity was recorded in Table 1. From Figure $1(\mathrm{a}, \mathrm{b})$, it is obvious that PLA has not exhibited any melting peak whereas, PHBV exhibited two melting peaks. The double peaks of PHBV may be due to the remelting and reorganization of imperfect crystal structure at higher temperature to crystalize with higher structural perfection [20]. PLA/ PHBV blend with different weight ratios showed mixed behavior.

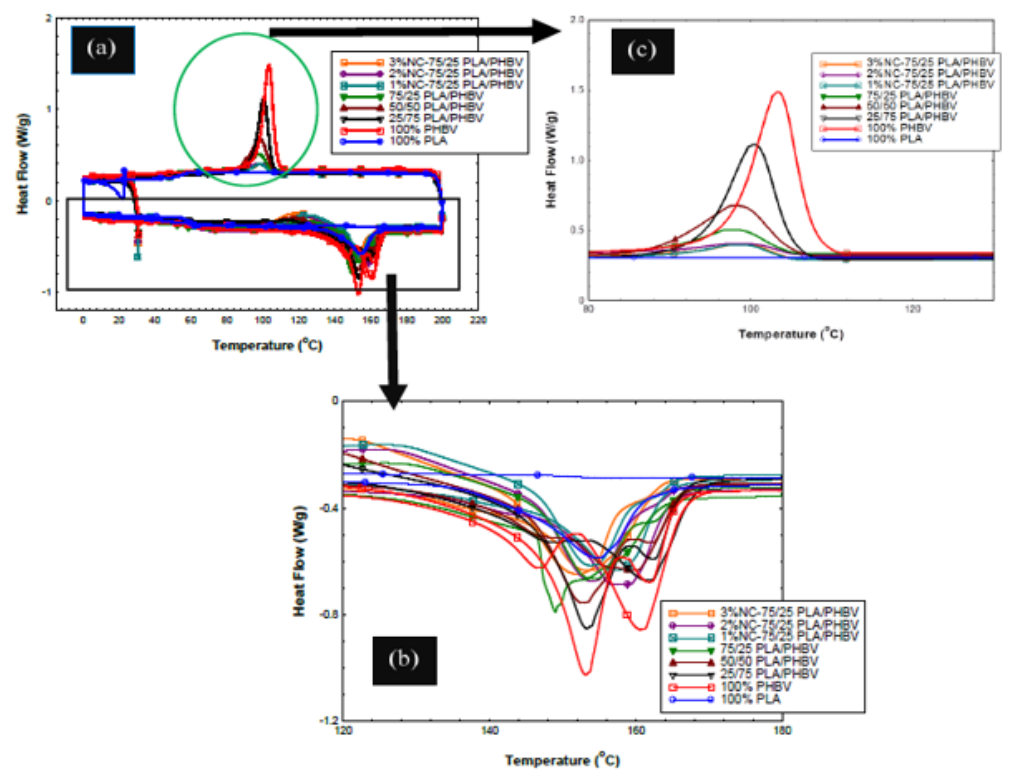

Figure 1: DSC curve of (a) PLA/PHBV blend with and without nano-clay, (b) Heating scan, and (c) Cooling scan of PLA/PHBV blend.

Table 1: Differential Scanning Calorimetry data of different PLA/PHBV blend with/ without nanoclay.

\begin{tabular}{|c|c|c|c|c|}
\hline Samples & $\Delta \mathrm{Hm}(\mathrm{J} / \mathrm{g})$ & $\operatorname{Tc}\left({ }^{\circ} \mathrm{C}\right)$ & $\operatorname{Tm}\left({ }^{\circ} \mathrm{C}\right)$ & Xc (\%) PHBV \\
\hline $100 \%$ PLA & $21.42 \pm 0.78$ & - & - & - \\
\hline $100 \%$ PHBV & $24.34 \pm 1.18$ & $114.05 \pm 0.335$ & $159.74 \pm 0.89$ & 16.7 \\
\hline 25\% PLA- 75\%PHBV & $36.45 \pm 1.58$ & $111.87 \pm 0.122$ & $160.11 \pm 0.231$ & 33.3 \\
\hline $50 \%$ PLA-50\% PHBV & $31.28 \pm 0.614$ & $109.71 \pm 0.95$ & $159.22 \pm 0.375$ & 42.9 \\
\hline 75\% PLA-25\% PHBV & $26.94 \pm 0.975$ & $109.05 \pm 0.578$ & $154.49 \pm 0.978$ & 73.8 \\
\hline 75\% PLA-25\% PHBV+1\%Nanoclay & $26.98 \pm 0.012$ & $109.23 \pm 0.0432$ & $155.34 \pm 0.056$ & 73.9 \\
\hline 75\% PLA-25\% PHBV+2\%Nanoclay & $27.10 \pm 0.187$ & $110.51 \pm 0.995$ & $157.24 \pm 0.159$ & 74.3 \\
\hline 75\% PLA-25\% PHBV+3\%Nanoclay & $23.89 \pm 0.079$ & $110.02 \pm 0.0672$ & $151.91 \pm 0.875$ & 65.5 \\
\hline
\end{tabular}


Several research groups reported that PLA, PHBV, and their copolymer show multiple melting peaks under isothermal crystallization during step scan DSC. Multiple melting peaks may be due to the remelting, recrystallization, isodimorphism or polymorphism (presence of multiple crystal form), different lamellar characteristics, and different molecular weight species [10]. The DSC curves of PLA/PHBV blend with and without nanoclay showed a large melting peak followed by a shoulder-like peak for residual crystals of PHBV. For a polymer blend, it is well established that the polymer with the higher melting temperature forms crystal before the polymer with lower melting temperature. But, the polymers with similar melting temperatures undergo co-crystallization to form another phase which acts as a heterogenous nucleation agent to produce prefect crystal structures during the melting process. In this research, both polymers have melting points around $160^{\circ} \mathrm{C}$.
Table 1 shows the degree of crystallinity of PLA, PHBV, PLA/ PHBV blend with and without nanoclay. Degree of crystallinity (DOC) of all polymers and blend was calculated by Eq. (1). The $\Delta \mathrm{Ho}$ value for PLA is 93.7 (J/g) which indicates that PLA cannot recrystallize (Figure 1(c)) at this heating rate due to slow crystallization and nucleation rates. DOC of PHBV was calculated in this study by considering PLA as a fully amorphous material. From Table 1, it can be inferred that DOC in PLA/PHBV increased with the increasing percentages of PLA. It may be due to the action of PLA as a nucleating agent [21]. This enhancement of DOC has a positive effect which would be reflected in mechanical property improvement. Furthermore, the addition of nanoclay with PLA/PHBV polymer blends influenced the DOC noticeably. The enhancement of DOC with the addition of nanoclay may be due to the good dispersion of nanoclay on polymer matrices which will be presented in SEM micrograph.

\section{Thermogravimetric analysis (TGA)}
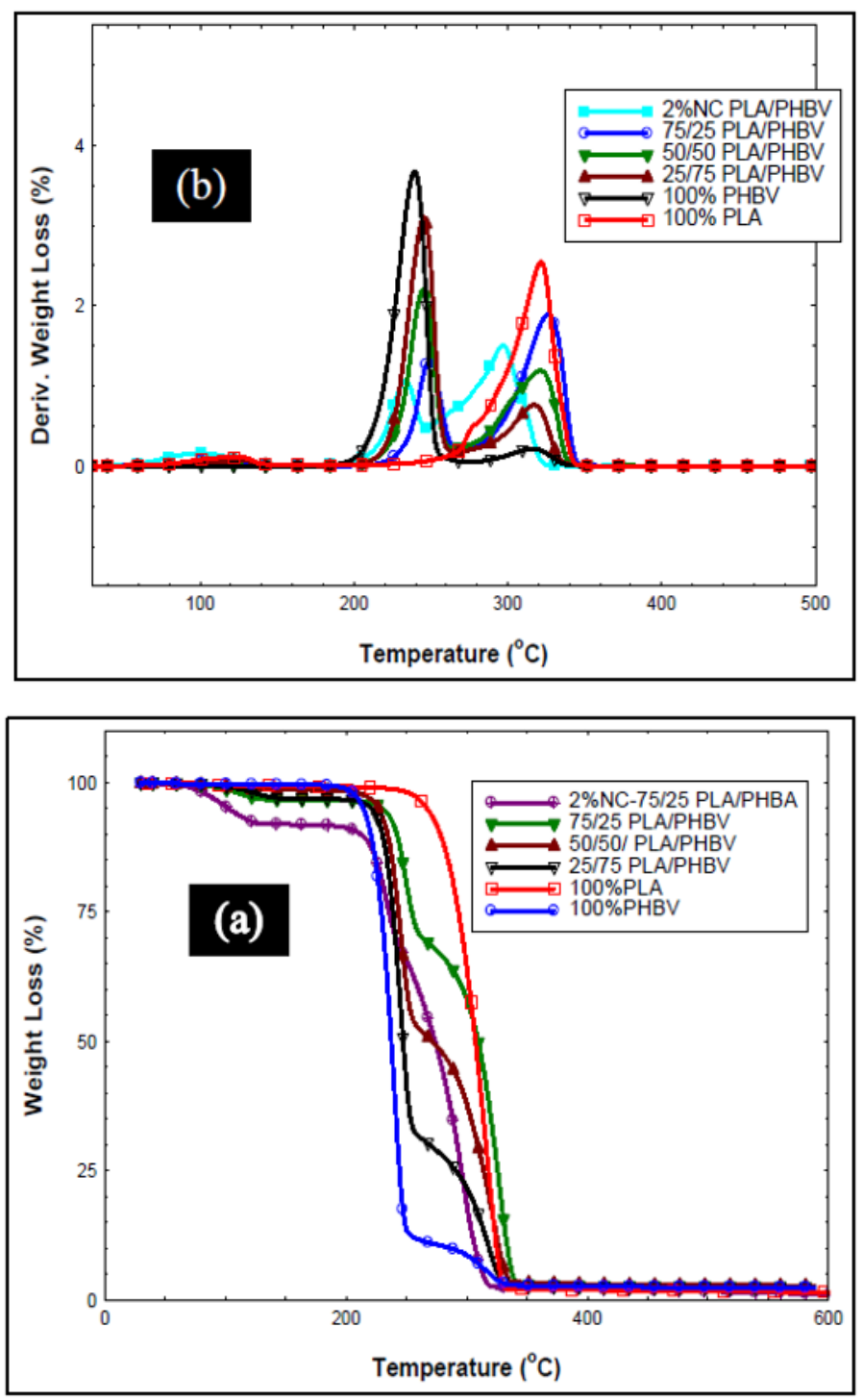

Figure 2: (a) TGA and (b) DTG graph of PLA/PHBV blend with and without nanoclay. 
Table 2: TGA and DTG data of different weight ratios PLA/PHBV blend.

\begin{tabular}{|c|c|c|c|c|}
\hline Name of the blend & T5\% $\left({ }^{\circ} \mathbf{C}\right)$ & T50\% $\left({ }^{\circ} \mathbf{C}\right)$ & Tp $\left({ }^{\circ} \mathbf{C}\right)$ & Residue Content $(\%)$ \\
\hline 100\% PLA & $284.51 \pm 0.069$ & $325.45 \pm 0.97$ & $315.39 \pm 6.67$ & $2.154 \pm 0.0975$ \\
\hline 100\% PHBV & $222.94 \pm 0.0871$ & $236.05 \pm 1.17$ & $239.88 \pm 3.2265$ & $2.049 \pm 0.1223$ \\
\hline 25\%PLA-75\%PHBV & $233.45 \pm 0.1167$ & $244.51 \pm 4.92$ & $245.053 \pm 0.9576$ & $2.20 \pm 0.113$ \\
\hline 50\%PLA-50\%PHBV & $234.58 \pm 0.156$ & $272.54 \pm 0.57$ & $245.82 \pm 0.79674$ & $2.40 \pm 0.695$ \\
\hline 75\%PLA-25\%PHBV & $240.16 \pm 0.77$ & $309.35 \pm 0.5467$ & $249.36 \pm 1.40$ & $2.554 \pm 0.785$ \\
\hline
\end{tabular}

Figure $2(a, b)$ represents the TGA and DTG curves of PLA/PHBV blend with three different compositions, including the optimized blend (75/25 wt.\%) with 2 wt.\% nanoclay based on DSC results. Thermogravimetric analysis was carried out under nitrogen atmosphere. From Figure 2 (a) it can be inferred that PLA and PHBV both showed single stage thermal degradation. But PLA started to degrade at $284.5{ }^{\circ} \mathrm{C}$, whereas PHBV degraded at $222.94{ }^{\circ} \mathrm{C}$ (Table 2). This slower degradation indicates higher thermal stability of PLA compared to PHBV. For any composition of PLA/PHBV blend, multistage thermal degradation was observed which can be attributed to the degradation of PHBV and PLA respectively. Multistage thermal degradation of all the blends were between PLA and PHBV which signifies the thermal stability of the blends was lower than PLA, but, higher than PHBV. DTG curves indicate the temperature at which maximum weight loss occurs [22]. From Figure 2(b), two sharp thermal degradation peaks were observed (Figure 2(b)) which can be attributed to the thermal degradation of PLA and PHBV components of the blend compared to the individual neat polymer. Maximum thermal degradation temperature of PLA component in the blend decreases with the increasing amount of PHBV, whereas, in the PHBV component, maximum thermal degradation temperature does not change, or the change was insignificant (Table 2). Furthermore, residue content of the blend increased with the increased weight percentage of PLA which represents more char formation at the end of the thermal degradation process. Therefore, 75/25 wt.\% blend exhibits better fire-resistant behavior than the individual polymer. It was observed that the optimized polymer blend showed maximum residue content with the addition of $2 \mathrm{wt} . \%$ nanoclay.
It may be due to the formation of thermal barriers formed by the nanoclay that restricts heat transfer to the matrices [9].

\section{Tensile testing}

Tensile tests on PLA, PHBV, as well as PLA/PHBV blend were conducted according to ASTM D882-02. All data has been reported by considering averages of at least five to six tests. From the tensile test results, it is apparent that mechanical properties of PLA based on elastic modulus, tensile strength, and elongation at break were better than PHBV whereas PLA/PHBV blends exhibited mechanical properties higher than PHBV but lower or close to PLA (Table 3). Mechanical properties of PLA/PHBV blend decreased with the increasing amount of PHBV. This decreasing trend can be attributed to the weak polymer interface of PLA and PHBV as well as bubble formation which eventually acts as a stress concentrator that leads to failure $[20,23]$. The optimal blend composition to achieve similar elastic modulus, tensile strength, and elongation at break of PLA was found to be $75 / 25$ wt.\%. For this combination of the blend, tensile strength was 12\% lower than PLA but, 53\% higher than PHBV. Alternatively, elongation at break, and elastic modulus enhanced around $101 \%$ and $70 \%$, respectively compared to PHBV. It was observed that due to the nanoclay addition, mechanical properties of PLA/PHBV blend enhanced significantly. 2 wt.\% nanoclay improved tensile strength, elastic modulus, as well as elongation at break by $92 \%, 90 \%$ and $168 \%$, respectively compared to the PHBV. This may be due to the good dispersion of nanoclay into the matrices which will be reflected in SEM micrograph.

Table 3: Tensile test data of PLA/PHBV blend with and without nanoclay.

\begin{tabular}{|c|c|c|c|}
\hline Compositions PLA/PHBV/Nano-clay (\% wt) & Elastic Modulus (MPa) & Tensile Strength (MPa) & Elongation at Break (\%) \\
\hline $100 / 0 / 0$ & $449.2 \pm 10.5$ & $29.20 \pm 2.0$ & $17.6 \pm 0.3$ \\
\hline $0 / 100 / 0$ & $274.3 \pm 13.1$ & $16.79 \pm 1.2$ & $5.38 \pm 0.2$ \\
\hline $25 / 75 / 0$ & $312.2 \pm 5.1$ & $17.86 \pm 1.7$ & $5.25 \pm 0.3$ \\
\hline $50 / 50 / 0$ & $450.4 \pm 10.8$ & $23.35 \pm 1.1$ & $6.71 \pm 0.2$ \\
\hline $75 / 25 / 0$ & $463.7 \pm 7.3$ & $25.73 \pm 0.5$ & $10.80 \pm 0.1$ \\
\hline $75 / 25 / 1$ & $520.8 \pm 8.7$ & $27.67 \pm 1.3$ & $12.52 \pm 0.4$ \\
\hline $75 / 25 / 2$ & $688.3 \pm 4.5$ & $32.32 \pm 2.5$ & $14.40 \pm 0.3$ \\
\hline $75 / 25 / 3$ & $360.8 \pm 11.6$ & $16.5 \pm 0.8$ & $8.30 \pm 0.2$ \\
\hline
\end{tabular}

\section{Morphological study}

Figure $4(a, b)$ represent the SEM micrographs of 75/25 wt.\% PLA/PHBV blend without and with 2 wt.\% nanoclay, respectively.
While nanoclay and polymer matrix interaction is pertinent in improving material properties, chemical interactions were not an area of interest in this study; and therefore, only physical interactions 
were considered here. From Figure 4(b) it can be inferred that the small white particles (denoted by the red circle) are nanoclay which is well dispersed throughout the polymer blend. No visible agglomeration is observed after the addition of $2 \mathrm{wt} . \%$ nanoclay. Also, thermodynamically polymer blends are immiscible, so, it is expected to observe the multiphase morphology in SEM micrograph. From Figure 4(b), PLA and PHBV phase can be identified clearly. The darker rough surface indicates PLA phase whereas smooth gray surface indicates PHBV phase. Nanoclay can be dispersed or embedded into either phase based on molar mass, as well as crystallinity. In this study, it has been observed that PLA shows amorphous nature (Figure1 $(\mathrm{a}, \mathrm{c})$ ). Therefore, nanoclay may be diffused more into PLA matrix which is visible (presence of white particle).

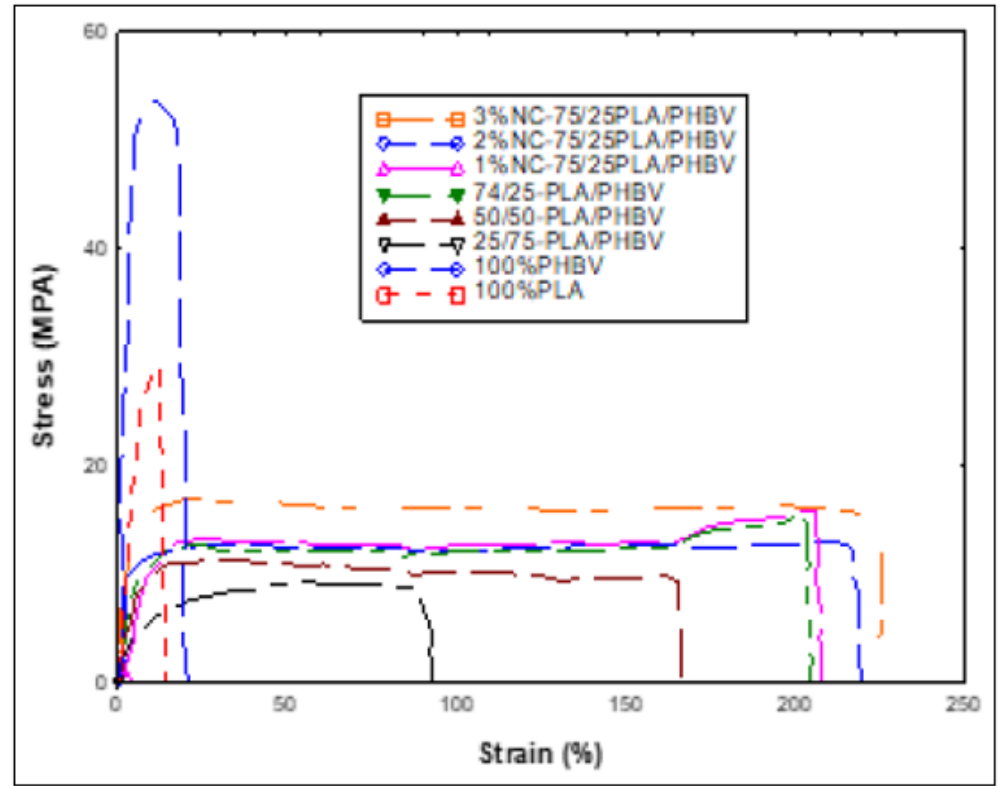

Figure 3: Stress-Strain graphs of PLA/PHBV blend with and without nanoclay.

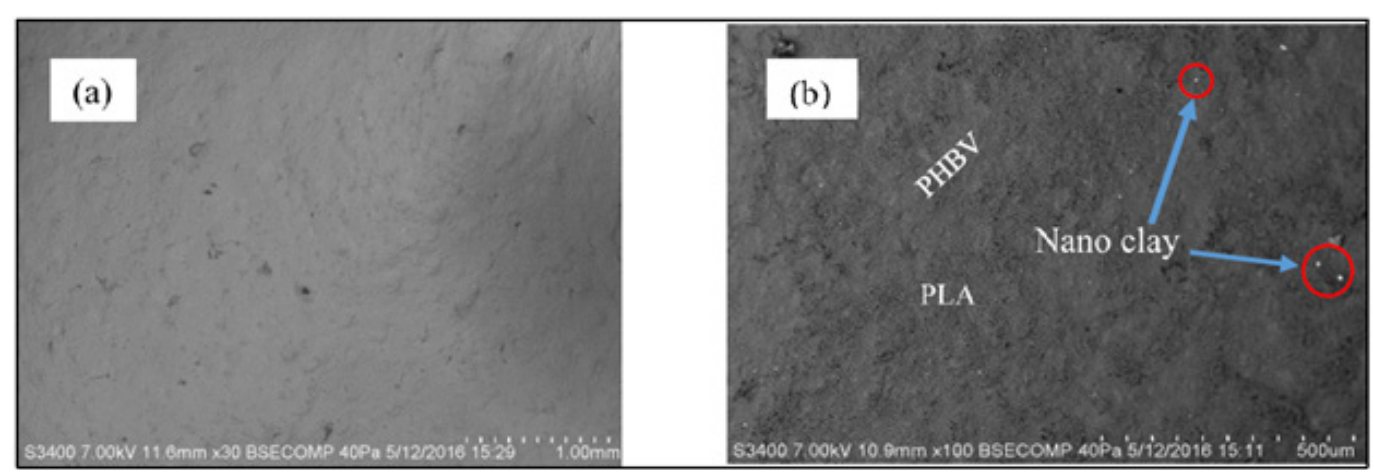

Figure 4: SEM image of (a) 75/25 wt. \% PLA/PHBV blend and (b) 75/25/Nanoclay blend at 500x magnification.

\section{Conclusion}

The results showed that the tensile strength of PLA thin film and PHBV film was found to be 29.20 MPa and 16.79 MPa, respectively. Hence, PLA has better mechanical properties than PHBV. On the other hand, thermal properties of PHBV thin film was found to be better than that of PLA. To optimize both mechanical and thermal properties of PLA and PHBV hybrid biopolymer blend, various combinations including 25/75, 50/50 and 75/25 wt.\% a hybrid biopolymer blend were prepared. Among them, PLA/PHBV (75/25 wt.\%) with 2 wt.\% nanoclay resulted in the best outcome. The tensile strength, elongation at break, and elastic modulus of this opti- mized polymer blend were $25.73 \mathrm{MPa}, 10.8$, and 463.7 MPa which are $53 \%, 101 \%$, as well as $70 \%$, respectively, higher than PHBV. From thermal analysis, two melting temperatures were observed in all the PLA/PHBV blend which represents solution immiscibility and melting of two polymer phases respectively. It had also been observed that the addition of nanoclay enhanced tensile properties as well as thermal stability up to $2 \mathrm{wt}$.\%. SEM micrographs showed rough surface and good dispersion of nanoclay throughout the polymer phases.

\section{Acknowledgement}

None. 


\section{Conflict of Interest}

No conflict of interest.

\section{References}

1. Mofokeng Julia P, Luyt Adriaan S (2015) Morphology and thermal degradation studies of melt-mixed PLA/PHBV biodegradable polymer blend nanocomposites with TiO2 as filler. J App Polym Sci 132(25).

2. Guan Qi Naguib, Hani E (2014) Fabrication and Characterization of PLA/ PHBV-Chitin Nanocomposites and Their Foams. J Polym. And the Env 22(1): 119-130.

3. Zhao Haibin, Cui Zhixian, Wang Xiaofeng, Turng Lih-Sheng, Penga Xiangfang (2013) Processing and characterization of solid and microcellular poly (lactic acid)/polyhydroxybutyrate-valerate (PLA/ PHBV) blends and PLA/PHBV/Clay nanocomposites. Comp Part B: Engineering 51: 79-91.

4. Corre YM, Maazouz A, Duchet J, Reignier J (2011) Batch foaming of chain extended PLA with supercritical $\mathrm{CO}_{2}$ : Influence of the rheological properties and the process parameters on the cellular structure. Supercrit Fluids 58(1): 177-188.

5. Lim LT, Auras R, Rubino M (2008) Processing technologies for poly (lactic acid). Prog Polym Sci 33(8): 820-852.

6. Pilla S, Kramschuster A, Yang LQ, Lee J, Gong SQ et al. (2009) Microcellular injection-molding of polylactide with chainextender. Mater Sci Eng C-Biomimetic Supramolec Syst 29(4): 1258-1265.

7. Hassan Eslami, Musa RK (2013) Elongational rheology of biodegradable poly [(lactic acid)/poly[(butylenesuccinate)-co-adipate] binary blends and poly (lactic acid)/poly [(butylene succinate) -co-adipate]/clay ternary nanocomposites. J Appl Polym Sci 127(3): 2290.

8. Richards E, Rizvi R, Chow A, Naguib H (2008) Biodegradable Composite Foams of PLA and PHBV Using Subcritical $\mathrm{CO}_{2}$. J Polym Environ 16(4): 258-266.

9. Hasan SMK, Zainuddin S, Tanthongsack J, Hosur MV, Allen L (2018) A study of poly (3-hydroxybutyrateco-3-hydroxyvalerate) biofilms' thermal and biodegradable properties reinforced with halloysite nanotubes. Joiournal of Composite Materials 52(23): 1-9.

10. Zhao Haibin, Cui Zhixiang, Sun Xiaofei, Turng Lih-Sheng, Peng Xiangfang (2013) Morphology and Properties of Injection Molded Solid and Microcellular Polylactic Acid/Polyhydroxybutyrate-Valerate (PLA/ PHBV) Blends. Ind and Eng Chem Res 52(7): 2569-2581.

11. Bledzki AK, Jaszkiewicz A (2010) Mechanical performance of biocomposites based on PLA and PHBV reinforced with natural fibres-A comparative study to PP. Compos Sci Technol 70(12): 1687-1696.
12. Si Mayu, Araki Tohru, Ade Harald, Kilcoyne ALD, Fisher Robert, et al. (2006) Compatibilizing Bulk Polymer Blends by Using Organoclays. Macromolecules 39(14): 4793-4801.

13. Elias L, Fenouillot F, Majeste JC, Cassagnauab PH (2007) Morphology and rheology of immiscible polymer blends filled with silica nanoparticles. Polymer 48(20): 6029-6040.

14. Laredo E, Grimau M, Bello A, Wu DF, Zhang YS, et al. (2010) AC Conductivity of Selectively Located Carbon Nanotubes in Poly( $\varepsilon$ caprolactone)/Polylactide Blend Nanocomposites. Biomacromolecules 11(5): 1339-1347.

15. Zainuddin S, Hasan SMK, Loeven Daniel, Hosur M (2019) Mechanical, Fire Retardant, Water Absorption, and Soil Biodegradation Profperties of Poly(3-hydroxy-butyrate-co-3-valerate) Nanofilms. J of Polym And the Env 27(10): 2292-2304.

16. Yuan MJ, Turng LS, Gong SQ Caulfield D, Hunt C, et al. (2004) Study of injection molded microcellular polyamide- 6 nanocomposites. Polym Eng Sci 44(4): 673-86.

17. Yuan MJ, Turng LS (2005) Microstructure and mechanical properties of microcellular injection molded polyamide- 6 nanocomposites. Polym 46(18): 7273-92.

18. Chandra A, Gong SQ, Yuan MJ, Turng LS, Gramann P, et al. (2005) Microstructure and crystallography in microcellular injection-molded polyamide-6 nanocomposite and neat resin. Polym Eng Sci 45(1): 52-61.

19. Mahdi TH, Islam Md E, Hosur MV, Jeelani S (2017) Low-velocity impact performance of carbon fiber-reinforced plastics modified with carbon nanotube, nanoclay and hybrid nanoparticles. J Rein Plas and Comp 36(9): 696-713.

20. Javadi A, Srithep Y, Lee J, Pilla S, Clemons C, et al. (2010) Processing and characterization of solid and microcellular PHBV/PBAT blend and its RWF/nanoclay composites. Compos Part a-Appl Sci Manuf 41(8): 982990.

21.Zhang M, Thomas NL (2011) Blending Polylactic Acid with Polyhydroxybutyrate: The Effect on Thermal, Mechanical, and Biodegradation Properties. Adv Polym Technol 30(2): 67-79.

22. Zhao Q, Wang SF, Kong MM, Geng WT, Li RKY, et al. (2012) Phase morphology, physical properties, and biodegradation behavior of novel PLA/PHBHHx blends. J Biomed Mater Res Part B-Appl Biomater 100B (1): 23-31.

23. Javadi A, Srithep Y, Pilla S, Lee J, Gong S, et al. (2010) Processing and characterization of solid and microcellular PHBV/coir fiber composites. Mater Sci Eng C-Mater Biol Appl 30(5): 749-757. 